\title{
La mouche et la libellule
}

De l'anachronisme en anthropologie des techniques

Pierre-Olivier Dittmar et Yann Philippe Tastevin

\section{(2) OpenEdition}

Journals

Édition électronique

URL : https://journals.openedition.org/tc/8463

DOI : $10.4000 /$ tc. 8463

ISSN : 1952-420X

Éditeur

Éditions de l'EHESS

\section{Édition imprimée}

Date de publication : 6 juin 2017

Pagination : 6-9

ISBN : 978-2-7132-2707-3

ISSN : 0248-6016

Référence électronique

Pierre-Olivier Dittmar et Yann Philippe Tastevin, « La mouche et la libellule », Techniques \& Culture [En

ligne], 67 | 2017, mis en ligne le 20 juin 2017, consulté le 29 septembre 2022. URL : http://

journals.openedition.org/tc/8463; DOI : https://doi.org/10.4000/tc.8463 


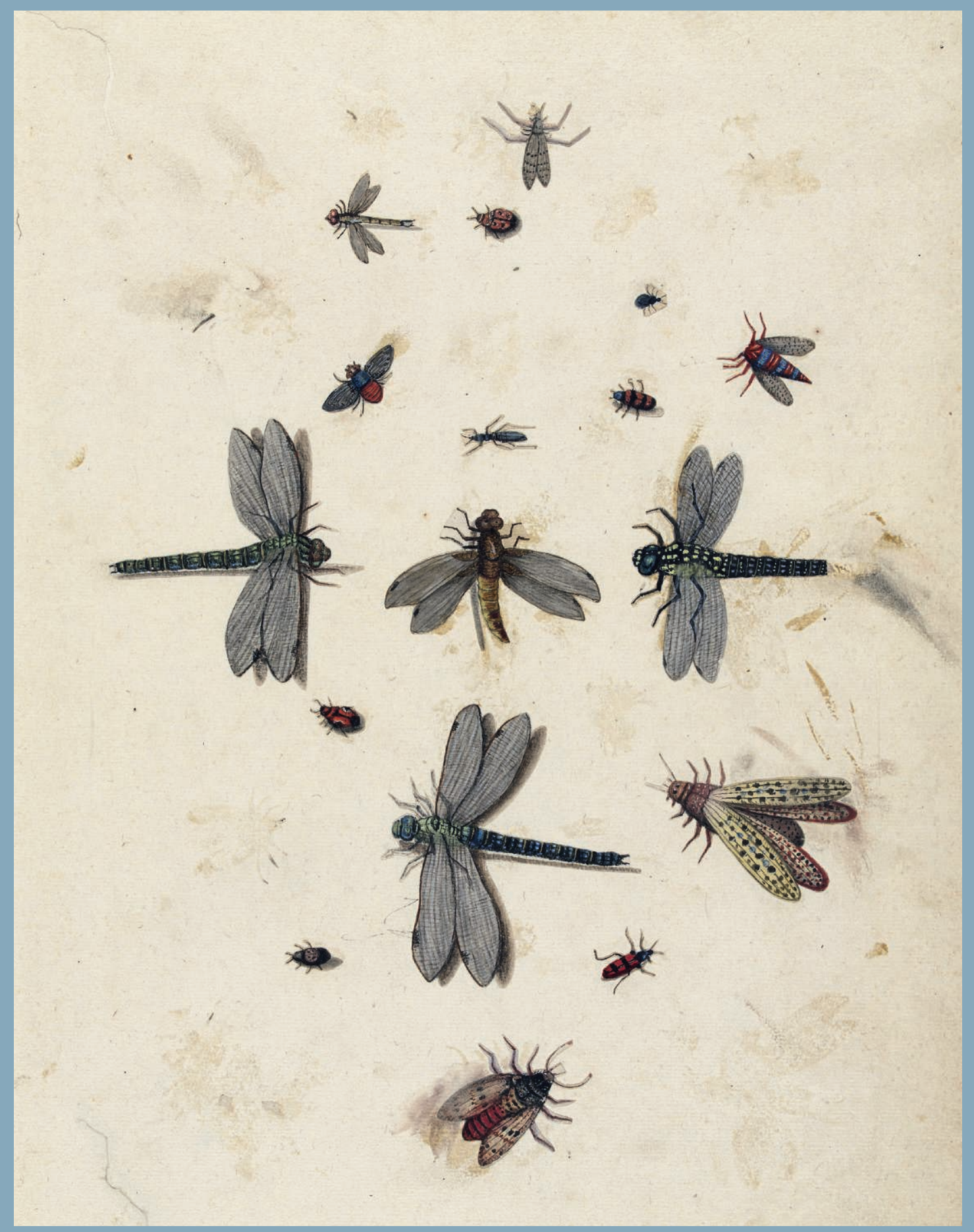




\section{Éditorial}

\section{La mouche et la libellule. De l'anachronisme en anthropologie des techniques}

Soit la libellule et la mouche. Les libellules font partie des plus vieux groupes d'insectes connus, les paléoptères. On en trouve les traces au dévonien et au carbonifère (entre 450 et 290 millions d'années). On peut a minima considérer que des spécimens proches de nos libellules actuelles vivaient au permien, il y a plus de 110 millions d'années, pour connaître depuis une remarquable stabilité. Dotées d'une morphologie archaïque (leurs ailes antérieures et postérieures sont indépendantes), les libellules ont un vol très peu spécialisé, leur permettant de voler sur place ou même en arrière. Performances « low tech » de la libellule.

En comparaison, les mouches, représentantes des diptères les plus fréquentés par les humains, font figure d'espèces « high tech ». Apparues bien plus récemment (il y a 65 millions d'années), avec leurs ailes postérieures transformées en altères et leur système de vision d'une incroyable sophistication, elles possèdent des caractéristiques remarquables et ont connu un succès incontestable au sein de la biodiversité : une espèce animale sur cinq est aujourd'hui un diptère.

Pour autant, les mouches savantes n'ont pas remplacé les libellules archaïques. Plus encore, la simplicité du mécanisme de ces dernières apparaît aujourd'hui comme une qualité précieuse si l'on se place du côté des usages plus que des structures. De fait, ces caractéristiques anachroniques présentent des possibilités d'appropriation exceptionnelles par les humains; elles font de la morphologie des libellules un terrain de réflexion privilégié pour les projets bio-inspirés, comme le micro-drone RENATA développé par l'Office national d'études et de recherches aérospatiales (ONERA) (Luc-Bouhali 2006, Seelow 2012).

Sans chercher à naturaliser les comportements sociaux, les destins parallèles de la mouche et de la libellule nous invitent à repenser la place que nous accordons en anthropologie des 
1. Machine de guerre, Giovanni Fontana v. $1420-1430$

techniques aux solutions «pauvres». Telle est bien l'ambition de ce numéro qui explore les conflits et les hybridations entre low tech et high tech. Dans le même temps, c'est aussi une question d'historicité posée au fil des articles de ce volume. Les pratiques low tech possèdent un rapport au temps singulier, déjouent les progressismes linéaires pour mieux interroger nos conceptions de l'évolution des techniques en intégrant la créativité propre aux usages. Elles invitent de fait à accorder dans nos disciplines une place aux vertus heuristiques de l'anachronisme et de l'exaptation ${ }^{1}$ (Lemonnier 2012), deux concepts qui ont déjà montré leur caractère opératoire dans d'autres domaines (Didi-Huberman 2000).

Après « Essais de bricologie » et « Réparer le monde », TechniquesE Culture achève avec ce numéro un triptyque visant à interroger à nouveaux frais l'anthropologie des techniques depuis ce qui apparaît comme les marges de la contemporanéité. Ces trois numéros visent chacun à leur manière à interroger la place des techniques dans les enjeux politiques contemporains marqués du triple sceau du capitalisme mondialisé, de l'urgence environnementale et de la dépossession des moyens de production. Cette enquête collective a été volontairement menée à partir de ce qu'on considérera comme un envers de ces phénomènes massifs en explorant en quoi, les artistes, les déchets, les techniques « pauvres » peuvent nous aider à repenser les évolutions actuelles et plus encore esquisser des solutions pour demain.

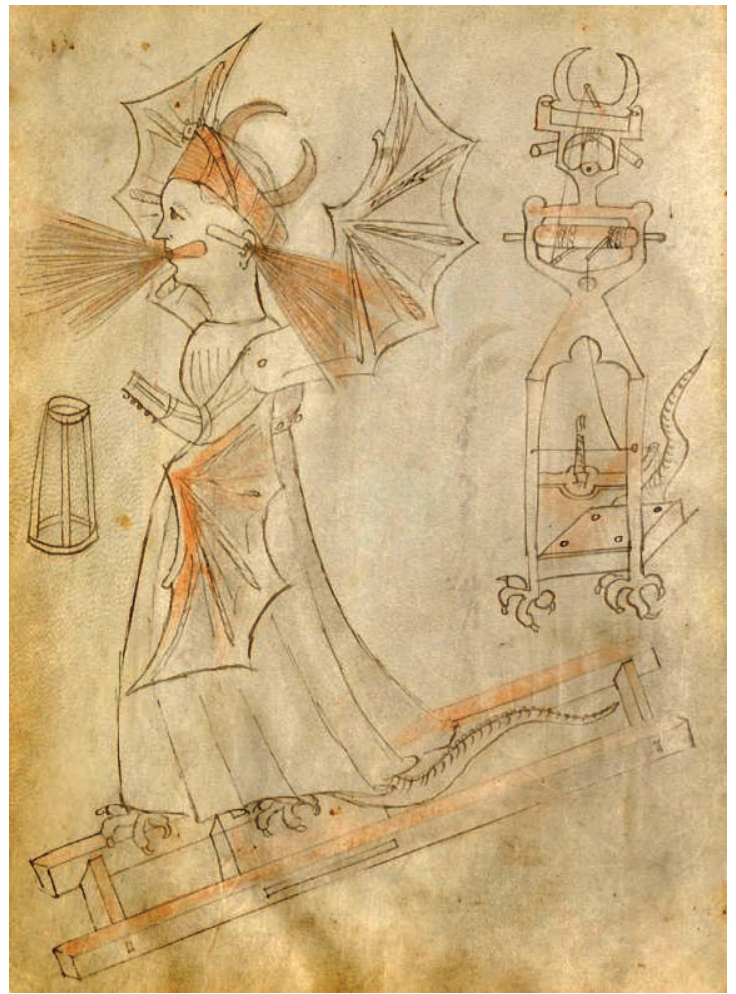

On connait le paradoxal procès en marginalité mené contre la low tech. D’une part les techniques pauvres ne seraient pas à même d'influer sur l'économie générale, elles seraient condamnées à rester la marque des "Suds», au mieux un pis-aller. D'autre part, les solutions low tech seraient l'expression d'un passe-temps utopique, un fantasme simultanément nostalgique et anecdotique réservé au contraire à ceux qui ont le luxe de ne pas se soumettre aux procès industriels les plus brutaux. Notre conviction est qu'une telle hiérarchisation doit être questionnée, et que les techniques sauvages offrent bien des solutions pour se débarrasser de certains dualismes stériles, comme celui qui oppose technophiles et technophobes, conflit chaque jour reconduit entre une technolâtrie basée sur la croyance dans un progrès continu et l'appel d'inspiration religieuse d'un retour à une création mythique pensée comme immuable et parfaite. Inscrites dans une philosophie du détournement plus que dans l'opposition catégorielle à l'innovation classique, les technologies « basses » ou « sauvages » se distinguent par les directions inattendues qu'elles donnent à l'innovation. La « wild tech » est ainsi un usage imprévu, inopiné d'une technologie qui redéfinit cette technologie. Que ce soit dans le temps ou dans les espaces, ces techniques qui apparaissent comme des alternatives sont plus que jamais à débusquer, inventorier et documenter. C'est 
précisément ce que Low-tech Magazine entreprend patiemment maintenant depuis une dizaine d'années (http://www.lowtechmagazine.com/low-tech-solutions.html). Ce journal en ligne, partenaire de Low Tech / High Tech, agrège une communauté de milliers de lecteurs et d'amateurs des techniques qui partagent nos préoccupations. Cette alliance s'inscrit dans la volonté durable de Techniques\&Culture de déborder l'espace des indispensables bibliothèques universitaires, pour exister en ligne, sur les réseaux sociaux, en librairie, dans l'espace public des festivals, rencontres ou musées. Une volonté de prendre simplement au sérieux le terme «publication ».

Idéalement les articles de ce numéro - et pourquoi pas ceux présents dans la revue depuis sa création - pourraient ainsi être vus comme un ensemble de ressources pratiques susceptibles un jour ou l'autre d'être réactivées. Ce serait la meilleure façon de rendre hommage au désir de Robert Cresswell qui rappelait dans un entretien publié en 1996, que « [le but de l'anthropologie] est double : d'une part l'étude doit conduire à une meilleure connaissance de nous-mêmes en tant qu'êtres sociaux, donc en fin de compte à une réflexion philosophique ; d'autre part cette connaissance doit aboutir à une action » (Bensa \& Cresswell 1996).

\section{Notes}

1. «La notion d'exaptation (exaptation) proposée par Gould et Vrba consiste à doter une forme ou un dispositif mécanique d'une fonction nouvelle et prometteuse, bien que sans grand rapport avec celle pour laquelle elle s'est (ou on l'a) initialement développée » P. Lemonnier, 2012.

\section{Iconographie}

Image d'ouverture. «Libellules et insectes volants », Lambert Lombard, vers 1560, h. $450 \mathrm{~mm} \times 1.315 \mathrm{~mm}$, Amsterdam, Rijksmuseum.

1. Giovanni Fontana, Bellicorum instrumentorum liber cum figuris - BSB Cod.icon. 242, Bl. 63v, v. 1420-1430.

\section{Références}

Bensa, A. \& R. Cresswell 1996 « À propos de la technologie culturelle. Entretien avec Robert Cresswell », Genèse 24 (1) : 121.

Didi-Huberman, G. 2000 Devant le temps. Histoire de l'art et anachronisme des images. Paris : Éditions de Minuit.

Lemonnier, P. 2012 « Fangio, Rozanoff et l'Espadon. À propos d'objets et d'imaginaires partagés » in J. Giraud \& G. Gernez dir. Aux marges de l'archéologie : 85-99.
Luc-Bouhali, A. 2006 « Progress on the Remanta project on MAV with flapping wings », EMAV. Braunschweig : Germany.

Seelow, S. 2012 « De la libellule au microdrone : comment les insectes nous apprennent à voler », Le Monde, 18.01.2012 [En ligne] : http://www.lemonde.fr/ technologies/article/2012/01/18/de-la-libellule-aumicrodrone-comment-les-insectes-nous-apprennenta-voler_1625200_651865.html.

\section{Pour citer l'article}

Dittmar, P.-O. \& Y. P. Tastevin 2017 «Éditorial. La mouche et la libellule. De l'anachronisme en anthropologie des techniques», Techniques\&Culture 67 « Low tech? Wild tech! », p. 6-9. 